\title{
Répteis em fragmentos de Cerrado e Mata Atlântica no Campo das Vertentes, Estado de Minas Gerais, Sudeste do Brasil
}

\author{
Bernadete Maria de Sousa ${ }^{1,2,5}$, Ana Elisa Resgalla do Nascimento ${ }^{1,2}$, Samuel Campos Gomides ${ }^{1,2}$, \\ Celso Henrique Varela Rios ${ }^{1,2}$, Alexandre de Assis Hudson ${ }^{4}$ \& Iara Alves Novelli ${ }^{1,3}$ \\ ${ }^{1}$ Laboratório de Herpetologia, Departamento de Zoologia, \\ Instituto de Ciências Biológicas, Universidade Federal de Juiz de Fora - UFJF, \\ Campus Universitário, s/n, CEP 36036-900, Martelos, Juiz de Fora, MG, Brasil \\ ${ }^{2}$ Programa de Pós-graduação em Biologia e Comportamento Animal, Instituto de Ciências Biológicas, \\ Universidade Federal de Juiz de Fora - UFJF, CEP 36036-900, Juiz de Fora, MG, Brasil \\ ${ }^{3}$ Laboratório de Zoologia, Centro Universitário de Lavras - UNILAVRAS, \\ Rua Padre José Poggel, 506, CEP 37200-000, Lavras, MG, Brasil \\ ${ }^{4}$ Centro Nacional de Pesquisa e Conservação de Répteis e Anfíbios - RAN, \\ Instituto Chico Mendes de Conservação da Biodiversidade - ICMBio, \\ Rua 229, 95, CEP 74605-090, Setor Leste Universitário, Goiânia, GO, Brasil \\ ${ }^{5}$ Autor para correspondência: Bernadete Maria de Sousa,e-mail: bernadete.sousa@ufjf.edu.br
}

SOUSA, B.M., NASCIMENTO, A.E.R., GOMIDES, S.C., VARELA RIOS, C.H., HUDSON, A.H. \& NOVELLI, I.A. Reptiles in fragments of Cerrado and Atlantic Forest at the Campo das Vertentes, Minas Gerais State, Southeastern Brazil. Biota Neotrop. 10(2): http://www.biotaneotropica.org.br/v10n2/en/ abstract?article+bn03510022010.

\begin{abstract}
Data on reptile fauna in the state of Minas Gerais are punctual and show the need of information about this group, mainly in transition regions between the Atlantic Forest and the Cerrado. The study area is located in Ritápolis municipality ( $21^{\circ} 01^{\prime} 37.07^{\prime}$ ' S and $\left.44^{\circ} 19^{\prime} 11.84^{\prime \prime} \mathrm{W}\right)$, micro-region Campo das Vertentes, State of Minas Gerais, Southeastern Brazil. The aim of this study was to study the composition of the local reptile fauna. The observations, captures and collections were performed every two weeks, during two consecutive days, from August 2005 to July 2006. The captures were made using pitfall traps with drift fences, divided in eight sites: four in the gallery forest area and four in open areas, totalizing 6.912 hours-bucket of sampling effort. We also performed active captures, used photographs to register occasional encounters and in some cases we also obtained snake specimens that were delivered by local people when found dead. We recorded a total of 31 reptile species, two turtle species; nine lizards; two amphisbaenians and 18 snakes. Only the lizards Cercosaura ocellata, Enyalius bilineatus and Tupinambis merianae and the snakes Leptodeira annulata and Apostolepis assimilis were captured by pitfall traps. The most common lizards were Ameiva ameiva and Mabuya frenata and the most abundant snakes were Oxyrhophus guibei and Sibynomorphus mikanii. Lizards are very well represented in the area, with species typical of the forest, as Enyalius bilineatus, and from Cerrado open areas, as Ameiva ameiva and Mabuya frenata. The snake assemblage contains species typical of Cerrado open areas (e. g. O. guibei and Micrurus frontalis), and of forests, as Liophis poecilogyrus and Philodryas olfersii. The diversity of reptile species and the previous record of Amphisbaena dubia and Hydromedusa tectifera in the state of Minas Gerais indicate the great potential of the Campo das Vertentes to reveal the occurrence of new species or to extend the distribution of other species.
\end{abstract}

Keywords: Testudines, Squamata, Ritápolis, Cerrado, Atlantic Forest.

SOUSA, B.M., NASCIMENTO, A.E.R., GOMIDES, S.C., VARELA RIOS, C.H., HUDSON, A.H. \& NOVELLI, I.A. Répteis em fragmentos de Cerrado e Mata Atlântica do Campo das Vertentes, Estado de Minas Gerais, Sudeste do Brasil. Biota Neotrop. 10(2): http://www.biotaneotropica.org.br/v10n2/pt/ abstract?article+bn03510022010.

Resumo: Os dados sobre a fauna reptiliana em Minas Gerais são pontuais e revelam carência de informações sobre esse grupo, principalmente em regiões de transição entre a Mata Atlântica e o Cerrado. A área do estudo

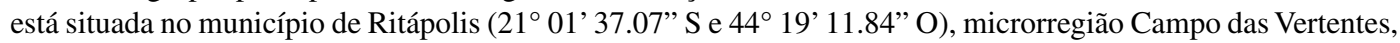
Estado de Minas Gerais, Sudeste do Brasil. Pretendeu-se com a presente pesquisa conhecer a composição da fauna de répteis local. As observações, capturas e coletas foram realizadas quinzenalmente, durante dois dias consecutivos, de agosto de 2005 a julho de 2006. As capturas foram realizadas por meio de armadilhas de interceptação e queda, distribuídas em oito sítios, sendo quatro em área de mata de galeria e quatro em área aberta, perfazendo um esforço amostral de 6.912 horas-balde. Foi também realizada procura ativa e encontros ocasionais com registros fotográficos dos espécimes, e, no caso de serpentes, alguns exemplares foram entregues por terceiros quando encontradas mortos. Registrou-se a presença de 31 espécies de répteis, sendo duas espécies de cágados, nove de lagartos, duas de anfisbenas e 18 de serpentes. Apenas os lagartos Cercosaura ocellata, Enyalius 
Sousa, B.M. et al.

bilineatus e Tupinambis merianae e as serpentes Leptodeira annulata e Apostolepis assimilis foram capturados nas armadilhas de queda. Os lagartos mais comuns foram Ameiva ameiva e Mabuya frenata, e as serpentes mais abundantes foram Oxyrhophus guibei e Sibynomorphus mikanii. Os lagartos estão bem representados na área, com espécies típicas de mata, como Enyalius bilineatus, e de áreas aberta de cerrado, como Ameiva ameiva e Mabuya frenata. A fauna de serpentes possui representantes típicos de áreas abertas do Cerrado, como O. guibei e Micrurus frontalis, e de regiões florestadas, como Liophis poecilogyrus e Philodryas olfersii. A diversidade de espécies de répteis e o registro prévio de Amphisbaena dubia e Hydromedusa tectifera para o Estado de Minas Gerais indicam a grande potencialidade do Campo das Vertentes em revelar a ocorrência de espécies novas ou a ampliação na distribuição de outras.

Palavras-chave: Testudines, Squamata, Ritápolis, Cerrado, Mata Atlântica.

\section{Introdução}

Os répteis formam um grupo proeminente em quase todas as taxocenoses terrestres, sendo conhecidas atualmente 9.084 espécies (Uetz \& Hallermann 2010). O Brasil ocupa atualmente a segunda posição em número de espécies (721; Bérnils 2010). No entanto, nos últimos anos, as populações de répteis têm sido vítimas de um declínio global tão sério quanto o de anfíbios. Tal declínio pode ser explicado por vários fatores, mas a perda e/ou degradação de hábitat adequado é a principal causa (Gibbons et al. 2000).

Entre os três biomas brasileiros com maior diversidade de répteis, a Amazônia abriga a maioria das espécies de serpentes, lagartos e anfisbenas, e o Cerrado e a Mata Atlântica se alternam em segundo lugar: o Cerrado tem a maior diversidade de lagartos e anfisbenídeos e a Mata Atlântica a maior diversidade de serpentes (Rodrigues 2005). Estudos visando determinar a composição da fauna de répteis de diferentes biomas do Brasil têm sido feitos, destacando-se os de Sazima \& Haddad (1992), Feio \& Caramaschi (2002), Marques \& Sazima (2004), Bertoluci et al. (2009), Palmuti et al. (2009) e Hartmann et al. (2009a, b) na Floresta Atlântica; Strüssmann \& Sazima (1993) no Pantanal; Colli et al. (2002), Vitt et al. (2002), Recoder \& Nogueira (2007) e Nogueira et al. (2009) no Cerrado, Vanzolini et al. (1980) na Caatinga, e Di-Bernardo (1998), Cechin (1999) e Zanella (2004) na Floresta de Araucária, Floresta Ombrófila Submontanhosa e nos campos da região Sul, respectivamente.

No Estado de Minas Gerais são encontrados os biomas Mata Atlântica, Cerrado e Caatinga, o que proporciona grande diversidade de anfíbios e répteis, favorecidos pela variedade de ambientes com diferentes formações vegetais e rochosas e sistemas hídricos, resultando também em um grande número de espécies endêmicas (Drummond et al. 2005). Mesmo com a riqueza de répteis existentes em Minas Gerais (31\% do total existente no Brasil), o nível de conhecimento sobre a fauna reptiliana no Estado é ainda insatisfatório e muito fragmentado (Drummond et al. 2005).

Embora a Mata Atlântica e o Cerrado sejam considerados os biomas mais ameaçados do planeta (Mittermeier et al. 2004), os estudos que caracterizam a composição da fauna reptiliana nestes biomas no Estado de Minas Gerais são escassos, e poucas são as informações sobre a biologia e o estado atual de conservação desse grupo, exceto pelos inventários realizados por Brites \& Bauab (1988), Assis (1999), Feio \& Caramaschi (2002), Recoder \& Nogueira (2007), Bertoluci et al. (2009), Costa et al. (2009), Palmuti et al. (2009) e São Pedro \& Pires (2009). A carência de informações sobre este grupo no estado dificulta a definição de áreas de preservação de maior interesse específico para a herpetofauna (Drummond et al. 2005).

Pretendeu-se com a presente pesquisa conhecer a composição da fauna de répteis do município de Ritápolis e ampliar o conhecimento sobre a herpetofauna em área de transição entre Cerrado e Mata Atlântica de Minas Gerais.

\section{Material e Métodos}

\section{1. Área de estudo}

O presente trabalho foi desenvolvido no Município de Ritápolis ( $21^{\circ} 01$ ' 37.07' S e 4419' 11.84' O), localizado na região Sudeste de Minas Gerais e Bacia do Rio Grande, mesorregião Campo das Vertentes e Serra das Vertentes. Trata-se de uma área de transição entre os biomas Mata Atlântica e Cerrado, situada entre as regiões mineiras da Zona da Mata, Sul de Minas e Centro-Oeste, com altitudes variando de 800 a $1300 \mathrm{~m}$ e temperatura média anual de $20^{\circ} \mathrm{C}$. A topografia, constituída por planaltos ondulados, é cortada por algumas elevações, tais como o Morro Grande e a Serra da Bandeira (PMR, 2006).

O bioma Cerrado foi considerado no sentido amplo, incluindo além das áreas abertas (desde campo limpo a cerradão e campos rupestres), formações florestais características (veredas, matas de galeria, matas mesofíticas). A área urbana do município está situada neste tipo de vegetação (Figura 1).

As armadilhas de interceptação e queda com uso de cerca guia foram instaladas em uma área de Cerrado e em uma mata de galeria da área de estudo, situada na área rural denominada Serra
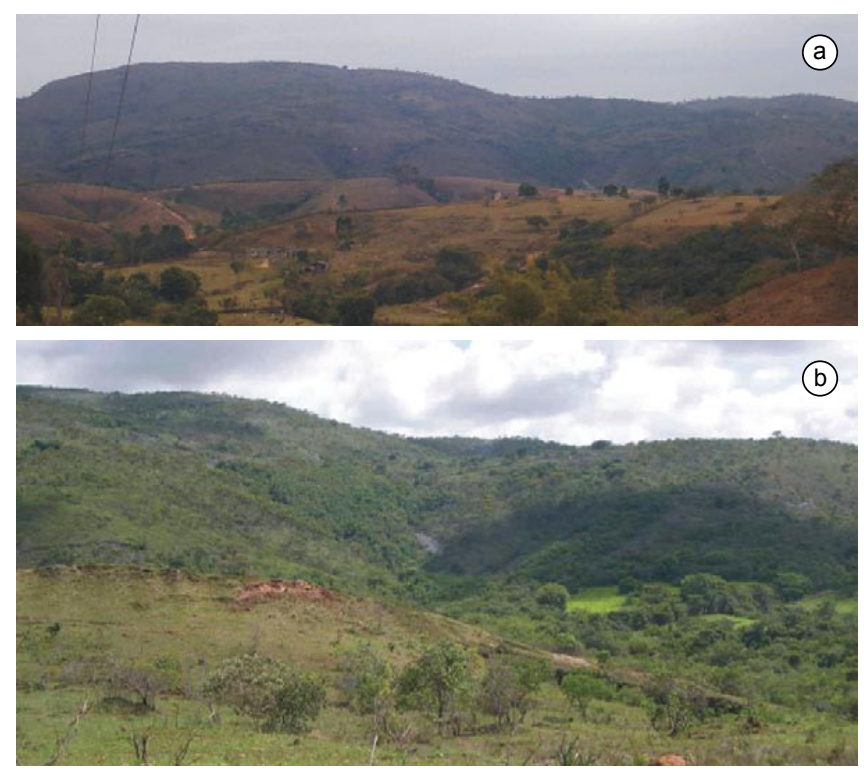

Figura 1. a) Vista da aérea urbana; b): fisionomia da área de estudo, localizada na Serra da Bandeira, no município de Ritápolis, Minas Gerais.

Figure 1. a) View of the urban area; b) the area of study (down), located in the Serra da Bandeira on the municipality of Ritápolis, Minas Gerais. 
( $21^{\circ} 00$ ' 02.42 " S e $44^{\circ} 18$ ' 16.09” O), localizada no município de Ritápolis, na Serra da Bandeira (Figura 2).

\section{Coleta de dados}

Os trabalhos de campo foram realizados mensalmente, no período de agosto de 2005 a julho de 2006, em excursões com duração de dois dias, totalizando 48 dias de campo. Para as capturas foram utilizados diferentes métodos de amostragem, conforme listados a seguir:

Armadilhas de interceptação e queda: Foram instalados quatro conjuntos de armadilhas de interceptação e queda com uso de cerca guia (pitfall traps with drift fences) como em Cechin \& Martins (2000), Sawaya et al. (2008) e Hartmann et al. (2009a, b). Nas áreas de cerrado e de mata foram instaladas quatro estações de coleta, cada uma composta de três linhas paralelas distantes $3 \mathrm{~m}$ uma da outra, com cinco baldes de $20 \mathrm{~L}$ em cada, sendo que as armadilhas de cada linha foram interligadas por uma cerca direcionadora de lona plástica preta com $50 \mathrm{~cm}$ de altura e enterradas $10 \mathrm{~cm}$ no chão. Em cada área, os sítios de coleta ficaram $40 \mathrm{~m}$ distantes um do outro, e cada conjunto distante $100 \mathrm{~m}$ do correspondente no outro trecho. Assim, foram instaladas 60 armadilhas em cada fitofisionomia, totalizando 120 armadilhas.

As armadilhas ficaram abertas durante quatro dias por mês, sendo vistoriadas no terceiro e no quarto dia. As inspeções foram sempre realizadas por, no mínimo, três pessoas. Caixas de plástico rígido com fechamento por trava foram utilizadas para o devido acondicionamento e transporte dos animais coletados a serem identificados.
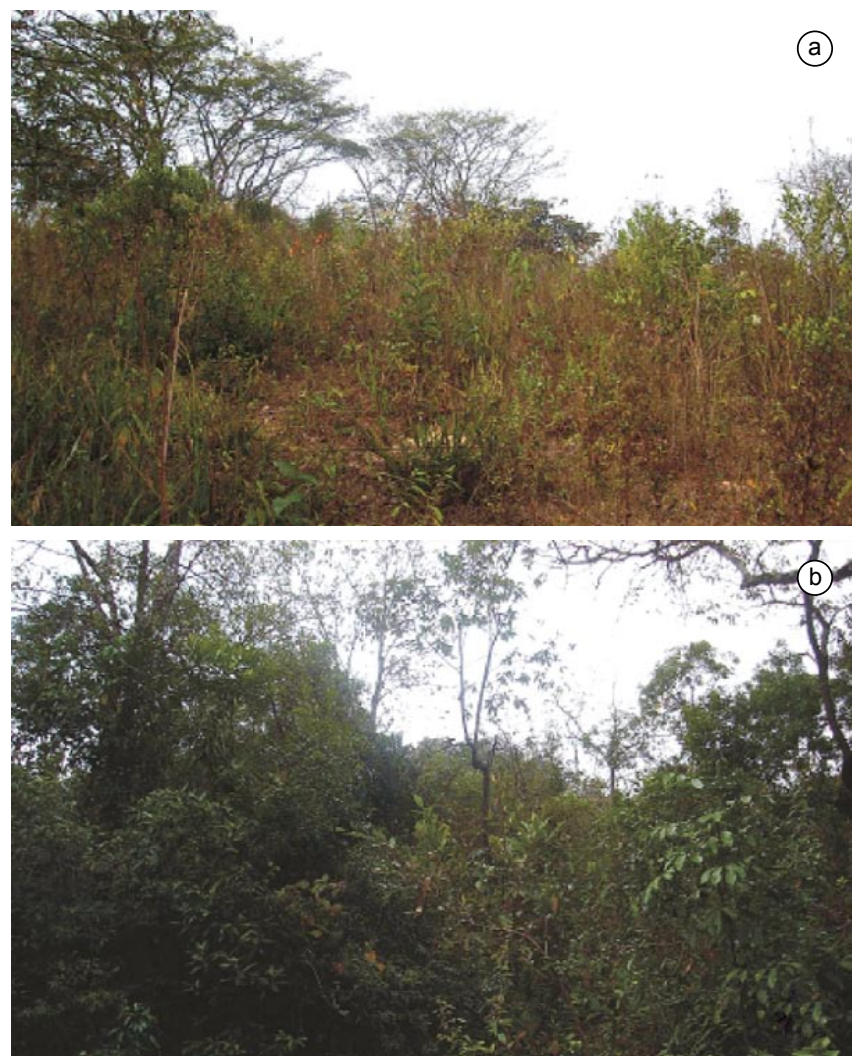

Figura 2. Áreas onde foram instaladas as armadilhas de interceptação e queda com uso de cerca guia; a) campo cerrado, b) borda da mata de galeria.

Figure 2. Areas where the pitfall traps with drift fences were installed: a) Cerrado field, b) Gallery forest.
Busca ativa: A busca ativa de répteis foi realizada quinzenalmente, durante dois dias consecutivos, com duração de 12 meses e preferencialmente nos períodos matutino (das 6:00 às 10:00) e vespertino (das 16:00 às 19:00). Os animais foram procurados em fendas de rochas, moitas de capim, embaixo de folhas, sobre pedras ao longo de córregos (Figura 3), ribeirões e brejos, em formigueiros e cupinzeiros, tocas no solo e galerias de roedores. $\mathrm{O}$ material utilizado para a procura constou de gancho, ancinho, palanca e puçá.

Além disso, foram considerados registros fotográficos decorrentes de encontros ocasionais e, no caso de serpentes, entrega por terceiros quando encontradas mortas. Dados de levantamentos da herpetofauna realizados entre os períodos de 04 a 08 de dezembro de 2002 e de 13 a 15 de dezembro de 2003 (B.M. Sousa, dados não publicados) também foram considerados neste inventário.

\section{Trabalho de laboratório}

No máximo três exemplares de cada espécie foram coletados e mortos com pentobarbital sódico, injetado na cavidade peritoneal. Sempre que possível, os indivíduos foram fotografados, pesados e medidos. Todos os indivíduos coletados foram depositados na Coleção Herpetológica da UFJF.

Para verificar a qualidade dos dados coletados e avaliar o esforço de coleta foram construídas curvas de acumulação de espécies a partir da média de 1.000 aleatorizações dos dias de coleta com o programa EstimateS V. 8.2.0, através do estimador Jackknife 2 (Colwell 2006).
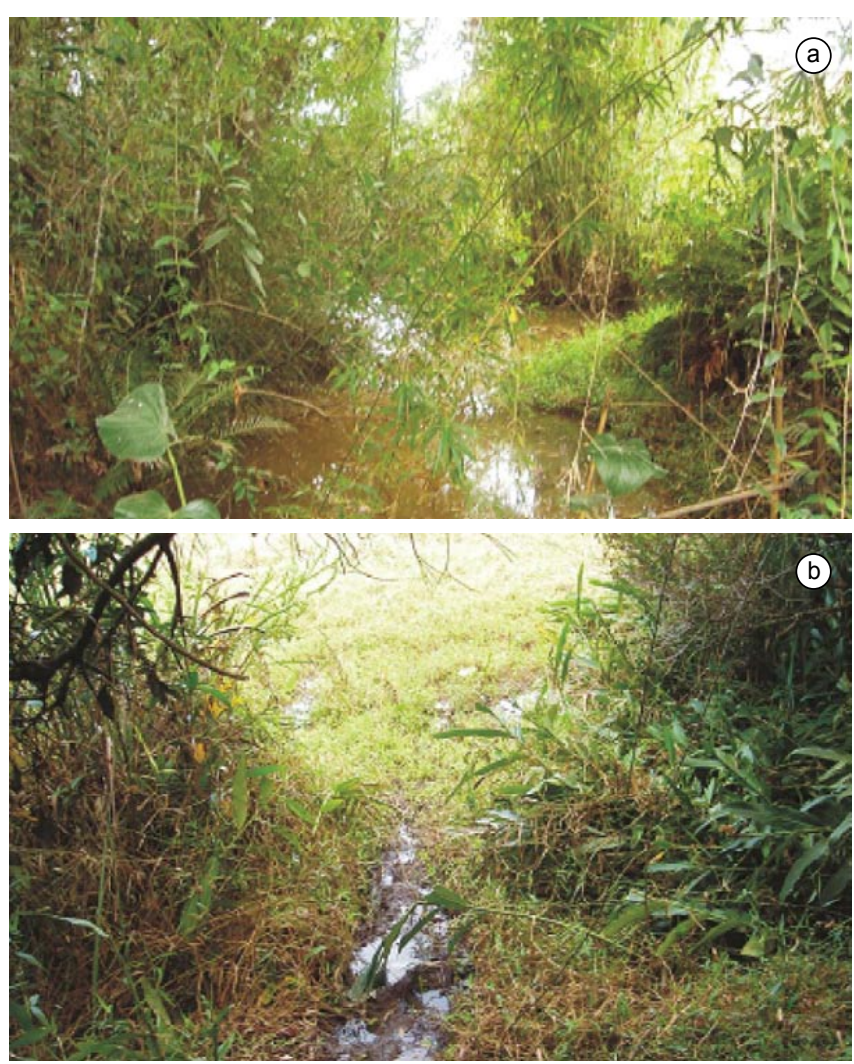

Figura 3. a-b) Ambientes aquáticos onde os espécimes de Testudines foram capturados.

Figure 3. a-b) Aquatic environments where the Testudines specimens were captured. 
O presente trabalho foi desenvolvido em consonância com os princípios adotados pelo COBEA (Colégio Brasileiro de Experimentação Animal) e aprovado pelo Comitê de Ética na Experimentação Animal da Pró-Reitoria de Pesquisa/UFJF e sob a licença do IBAMA.

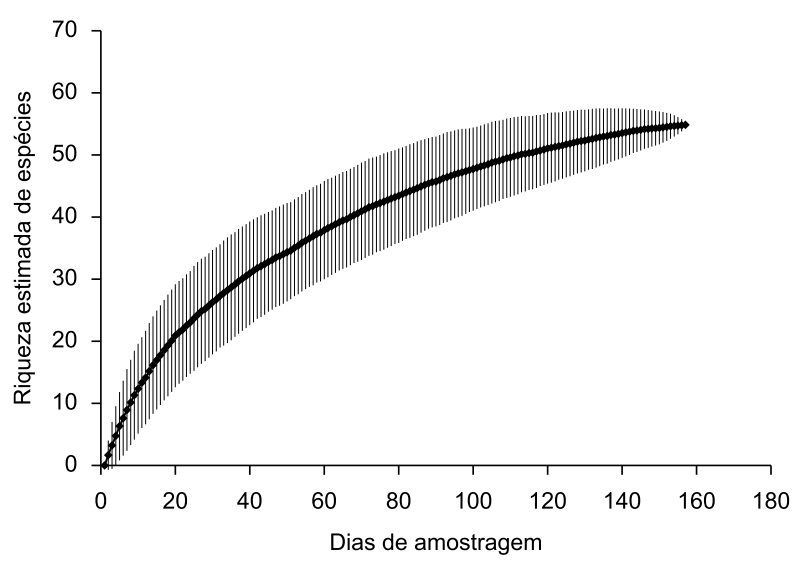

Figura 4. Curva de acumulação das espécies de répteis amostradas no Município de Ritápolis, Estado de Minas Gerais. As barras verticais representam os desvios padrão para os valores estimados.

Figure 4. Accumulation curve of the reptile species registered in Ritápolis municipality, state of Minas Gerais. Vertical bars represent standard deviation of the estimated values.

\section{Resultados}

Com um esforço amostral de 6.912 horas-balde e 384 horas-homem foi registrada a presença de 31 espécies de répteis. A curva de acumulação de riqueza estimada de espécies mostrou um rápido incremento no número de espécies conforme esforço amostral. Embora a curva se desloque em tendência à assíntota, pelo seu grau de inclinação, provavelmente mais espécies possam ser encontradas na área, uma vez que a assíntota plena não foi atingida (Figura 4). Com base no estimador Jackknife de segunda ordem, a riqueza estimada para a área de estudo é de aproximadamente 55 espécies.

Foram registradas duas espécies de quelônios da família Chelidae (Figura 5a-b), nove espécies de lagartos, distribuídas em oito famílias (Figuras 5c-h; 6a-d), duas espécies de anfisbenas da família Amphisbaenidae (Figura 6e-f) e 18 espécies de serpentes, incluídas em quatro famílias (Figuras 6g-h; 7a-h; 8a, b): Boidae (1 espécie), Dipsadidae (13), Elapidae (1) e Viperidae (3). Todas as espécies registradas, bem como os ambientes amostrados e a sua distribuição no Brasil estão na Tabela 1. Nenhuma das espécies se encontra nas listas de espécies ameaçadas de extinção do Brasil (Machado et al. 2008) ou de Minas Gerais (COPAM 2008).

As duas espécies de quelônios, o cágado-pescoço-de-cobra Hydromedusa tectifera (Figura 5a) e o cágado amarelo Acanthochelys radiolata (Figura 5b), foram encontradas nos ambientes aquáticos da área de estudo, em áreas de remanso e áreas brejosas, respectivamente, durante a busca ativa.
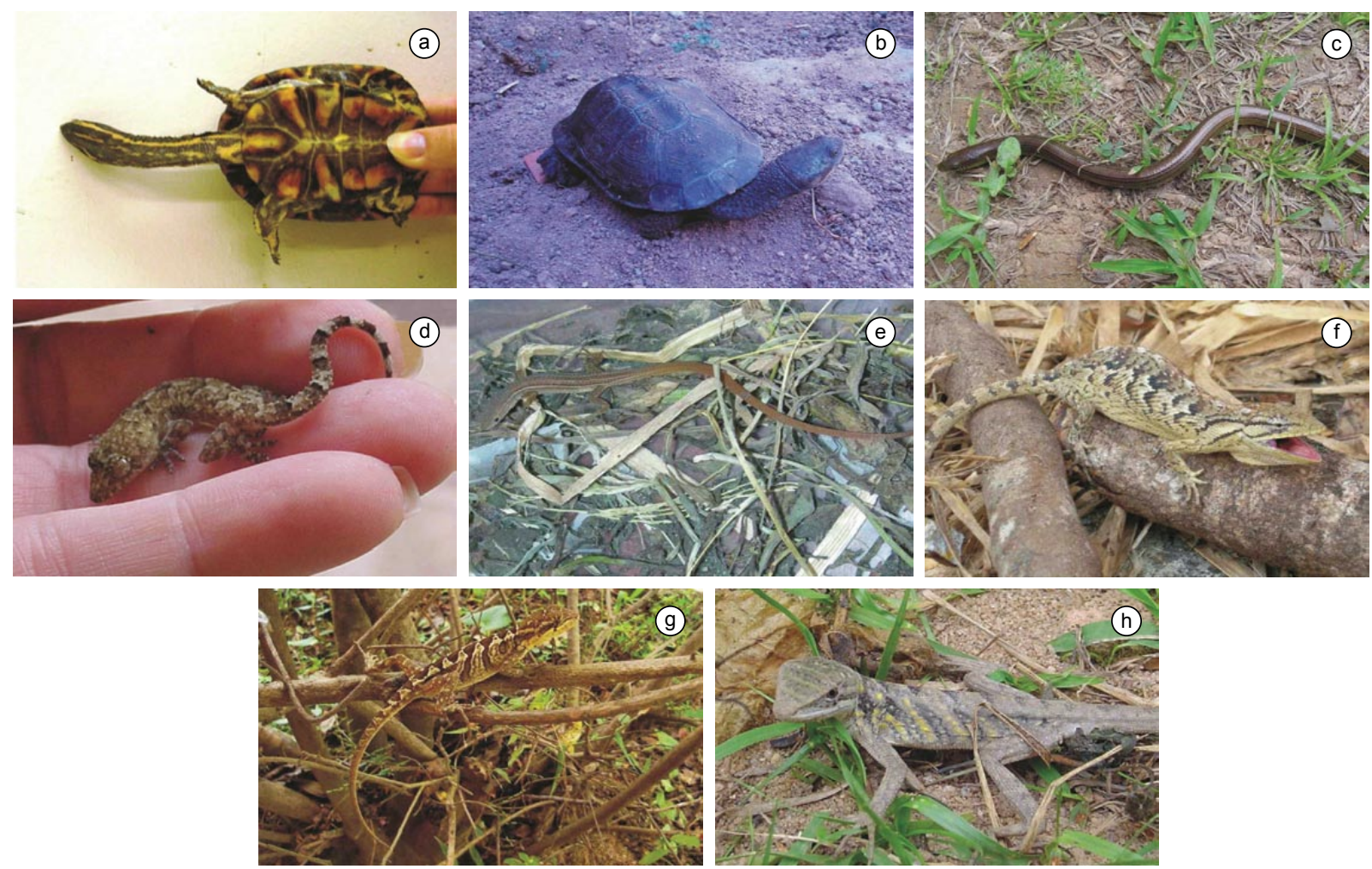

Figura 5. Algumas espécies de répteis registradas em fragmentos de Cerrado e Mata Atlântica do Campo das Vertentes, Estado de Minas Gerais, Sudeste do Brasil. a) Hydromedusa tectifera; b) Acanthochelys radiolata; c) Ophiodes striatus; d) Hemidactylus mabouia; e) Cercosaura ocellata; f) Polychrus acutirostris; g) Enyalius bilineatus fêmea; e h) Enyalius bilineatus macho.

Figure 5. Some species of reptiles recorded in the fragments of Cerrado and Atlantic Forest at the Campo das Vertentes, Minas Gerail State, Southeastern Brazil. a) Hydromedusa tectifera; b) Acanthochelys radiolata; c) Ophiodes striatus; d) Hemidactylus mabouia; e) Cercosaura ocellata; f) Polychrus acutirostris; g) Enyalius bilineatus female; and h) Enyalius bilineatus male. 

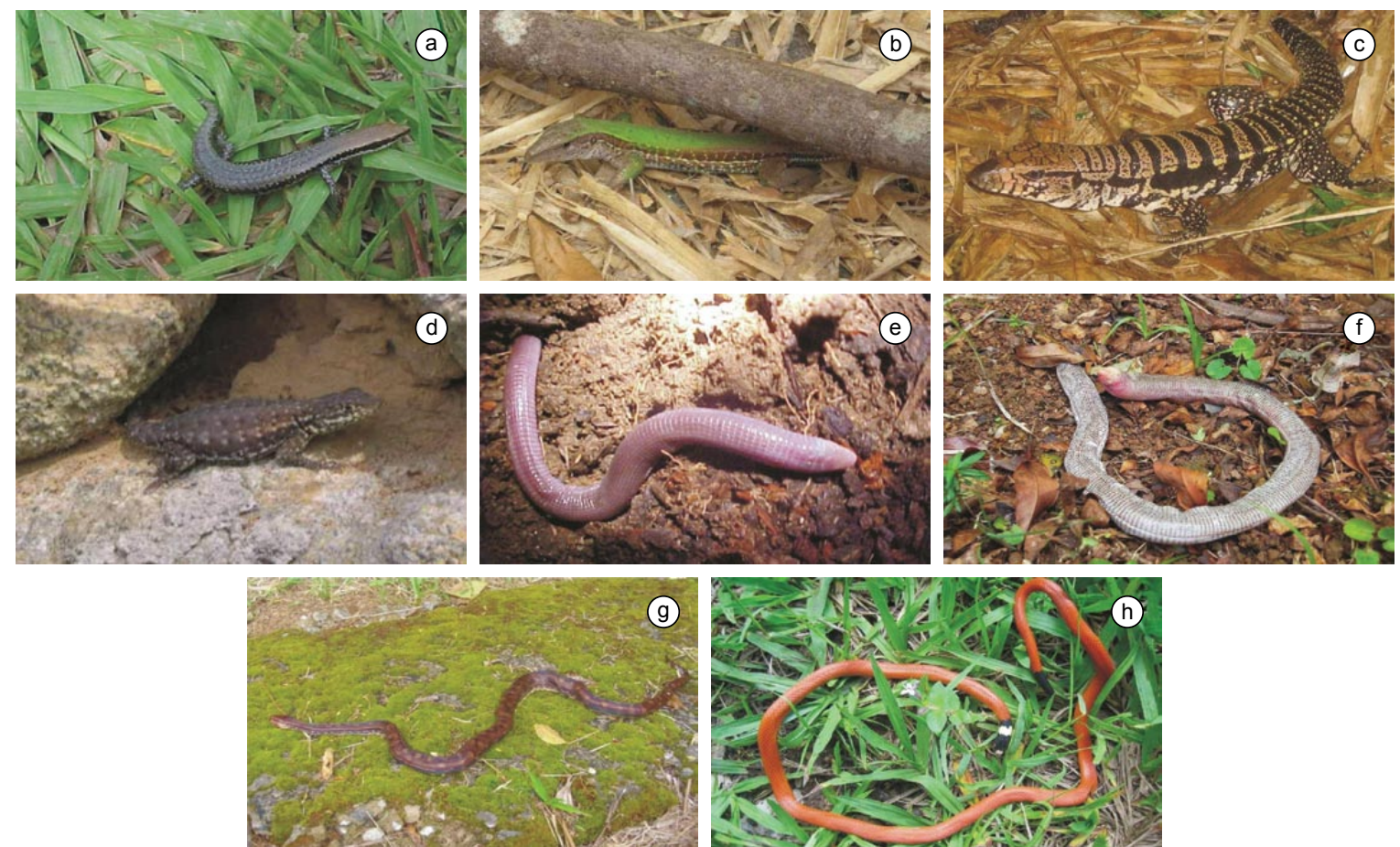

Figura 6. Algumas espécies de répteis registradas em fragmentos de Cerrado e Mata Atlântica do Campo das Vertentes, Estado de Minas Gerais, Sudeste do Brasil. a) Mabuya frenata; b) Ameiva ameiva; c) Tupinambis merianae; d) Tropidurus itambere; e) Amphisbaena dubia; f) Amphisbaena microcephala; g) Epicrates crassus; e h) Apostolepis assimilis.

Figure 6. Some species of reptiles recorded in the fragments of Cerrado and Atlantic Forest at the Campo das Vertentes, Minas Gerail State, Southeastern Brazil. a) Mabuya frenata; b) Ameiva ameiva; c) Tupinambis merianae; d) Tropidurus itambere; e) Amphisbaena dubia; f) Amphisbaena microcephala; g) Epicrates crassus; and h) Apostolepis assimilis.
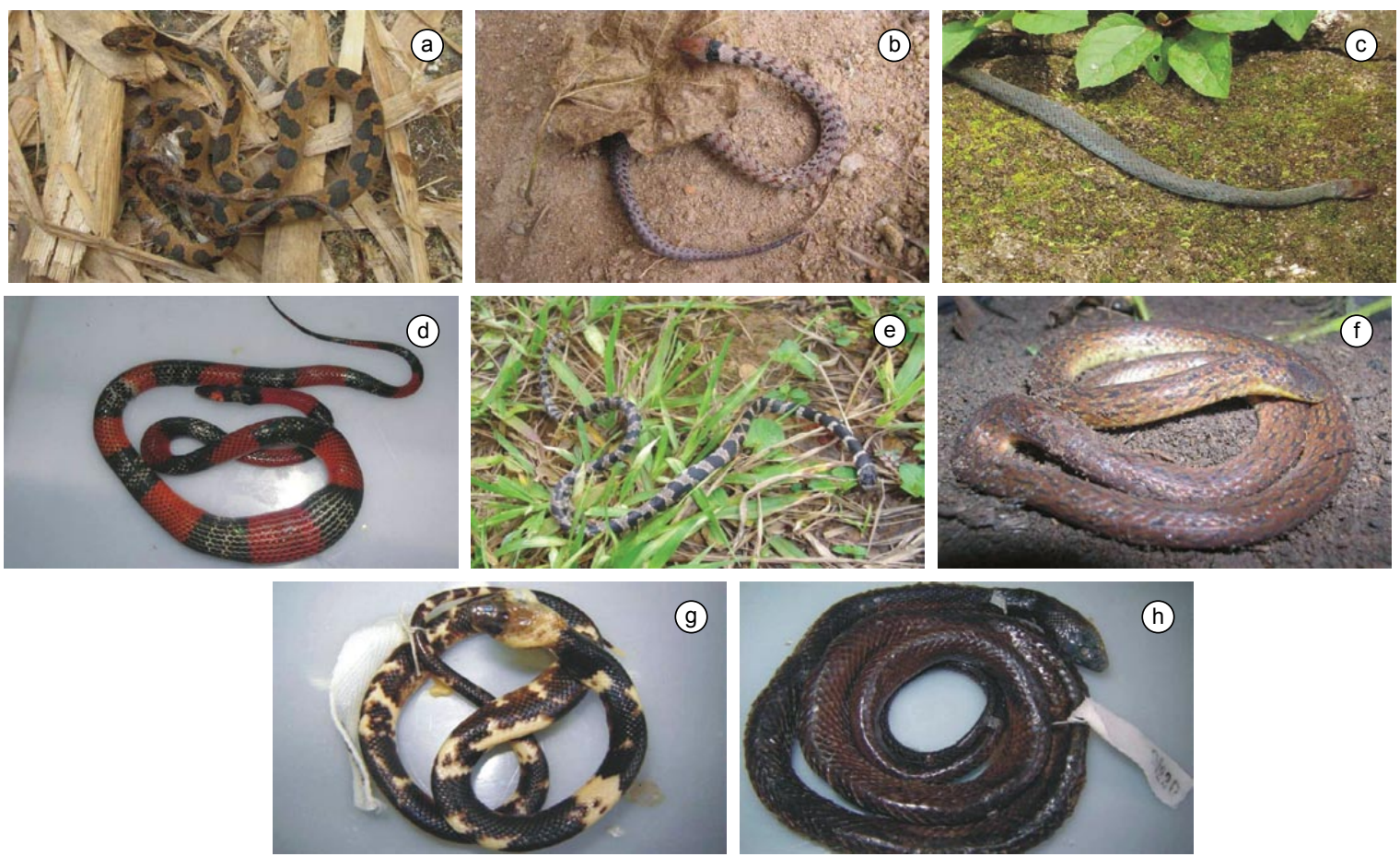

Figura 7. Algumas espécies de répteis registradas em fragmentos de Cerrado e Mata Atlântica do Campo das Vertentes, Estado de Minas Gerais, Sudeste do Brasil. a) Leptodeira annulata; b) Liophis poecilogyrus; c) Liophis typhlus; d) Oxyrhopus guibei; e) Sibynomorphus mikanii; f) Atractus pantostictus; g) Pseudoboa nigra; e h) Pseudoboa serrana.

Figure 7. Some species of reptiles recorded in the fragments of Cerrado and Atlantic Forest at the Campo das Vertentes, Minas Gerail State, Southeastern Brazil. a) Leptodeira annulata; b) Liophis poecilogyrus; c) Liophis typhlus; d) Oxyrhopus guibei; e) Sibynomorphus mikanii; f) Atractus pantostictus; g) Pseudoboa nigra; and h) Pseudoboa serrana. 

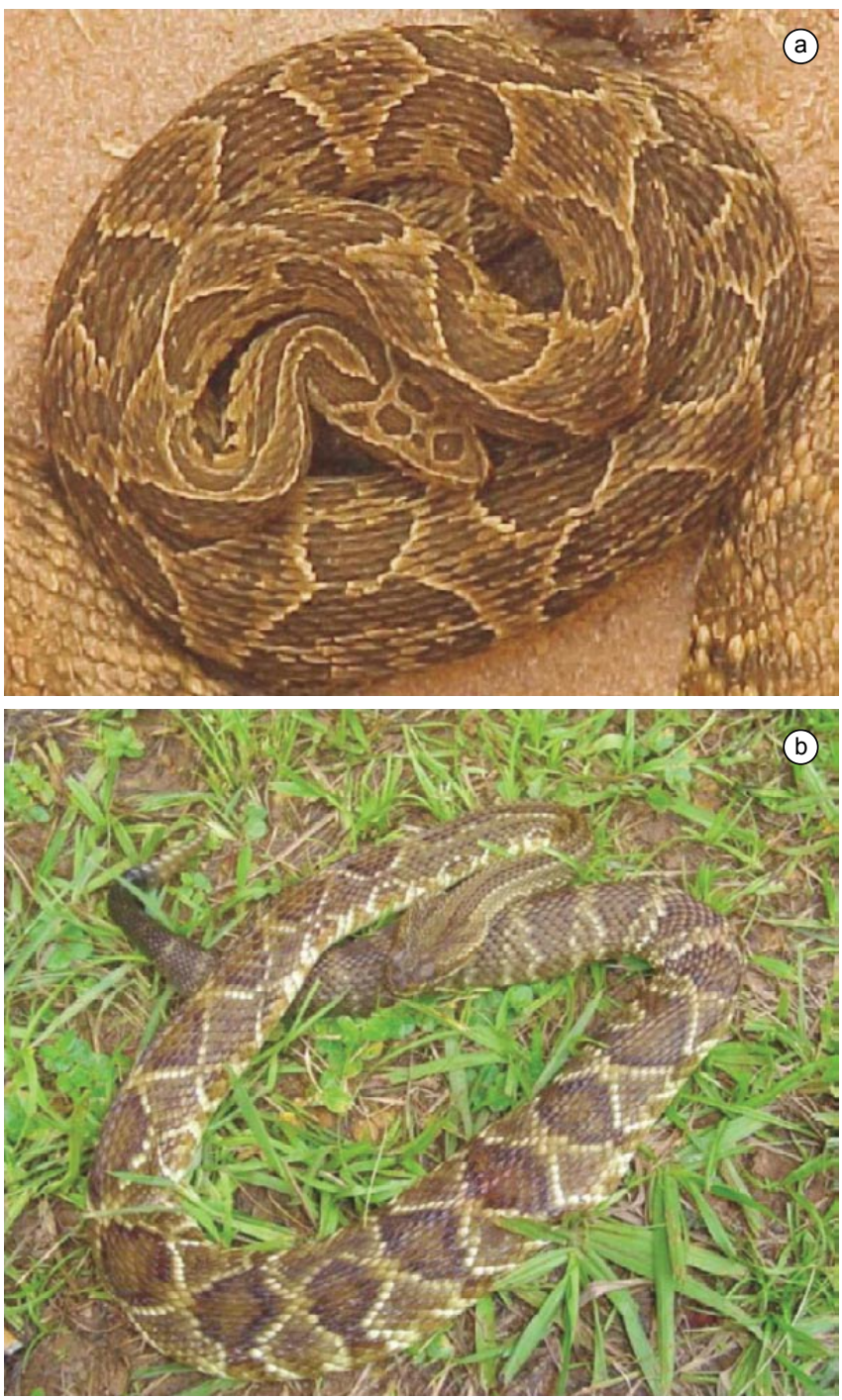

Figura 8. Algumas espécies de répteis registradas em fragmentos de Cerrado e Mata Atlântica do Campo das Vertentes, Estado de Minas Gerais, Sudeste do Brasil. a) Bothropoides neuwiedi; e b) Caudisona durissa.

Figure 8. Some species of reptiles recorded in the fragments of Cerrado and Atlantic Forest at the Campo das Vertentes, Minas Gerail State, Southeastern Brazil. a) Bothropoides neuwiedi; and b) Caudisona durissa.

Apenas os lagartos Cercosaura ocellata (Figura 5e), Enyalius bilineatus (Figura 5g-h) e Tupinambis merianae (Figura 6c) foram capturados exclusivamente nas armadilhas de queda. Os lagartos mais comuns nas armadilhas foram Enyalius bilineatus e Ameiva ameiva (Figura 6b), e os encontrados mais comumente por procura visual foram A. ameiva e Mabuya frenata (Figura 6a). A busca ativa possibilitou captura de indivíduos de seis $(67 \%)$ espécies de lagartos.

Com exceção de um espécime de Leptodeira annulata (Figura 7a) e um de Apostolepis assimilis (Figura 6h), que foram capturados em armadilhas, todos os outros exemplares de Atractus pantostictus (Figura 7f), L. annulata, Oxyrhopus guibei (Figura 7d), Sibynomorphus mikanii (Figura 7e) e Xenodon merremii foram coletados de forma ativa. Os demais espécimes destas e de outras espécies foram encontrados mortos, principalmente atropelados na via de acesso principal ao município. As serpentes mais abundantes foram $O$. guibei e S. mikanii.
Três exemplares de Epicrates crassus (Figura 6g) foram registrados para o município, sendo que um foi registrado por foto em uma área de Cerrado associado a matas de galeria da área de estudo, e dois outros foram encontrados mortos: um próximo à área de estudo e o outro na área urbana a aproximadamente $8 \mathrm{~km}$ da área de estudo.

\section{Discussão}

O número de espécies registradas no presente trabalho representa cerca de $15 \%$ do total de répteis estimado para o Estado de Minas Gerais segundo os dados de Bertoluci (1998) e 14\% em relação aos dados de Bérnils et al. (2009), embora deva estar subestimado, uma vez que muitas espécies esperadas para a região não foram encontradas. Muito provavelmente seja necessária a utilização de métodos mais eficientes e maior esforço de coleta para amostrar toda a fauna de répteis da região. Foi possível, contudo, a partir dos dados obtidos, estimar a composição da comunidade de répteis local, assim como seus hábitats preferenciais.

Os cágados das espécies $H$. tectifera e $A$. radiolata são espécies brasileiras de Chelidae, com distribuição na Mata Atlântica, exceto relato de Sousa \& Novelli (2009), que registraram essa espécie pela primeira vez no bioma Cerrado com esse espécime capturado nesse estudo. Acanthochelys radiolata, também conhecida como cágadoamarelo, distribui-se desde São Paulo, Rio de Janeiro, Minas Gerais, Espírito Santo, Bahia e Sergipe até Alagoas (Ernst \& Barbour 1989, Rodrigues 2005, Souza 2005, Fritz \& Havas 2007, Römpp 2008). É uma espécie que vive em pequenos lagos e lagoas de movimento lento e abundante vegetação aquática, com fundo macio onde pode se enterrar (Ernst \& Barbour 1989, Bonin et al. 2006).

Entre os anfisbenídeos, Amphisbaena microcephala (Figura 6f), foi registrada no presente estudo e relatada anteriormente em área de Cerrado em Brasília, DF (Nogueira 2001) e em área de Mata Atlântica no município de Viçosa, MG (Costa et al. 2009), sendo uma espécie de ampla distribuição no Sudeste do Brasil (Perez \& Ribeiro 2008). A ampliação na distribuição de Amphisbaena dubia (Figura 6e) para Minas Gerais foi registrada por Barros Filho et al. (2005), a qual anteriormente estava limitada aos Estados de São Paulo, Paraná e Santa Catarina. Até o presente momento, este continua sendo o único registro dessa espécie em Minas Gerais.

Com exceção de Hemidactylus mabouia (Figura 5d) e Tupinambis merianae, os demais lagartos também foram registrados para áreas do Cerrado no Brasil Central por Nogueira et al. (2009), incluindo o Parque Nacional Serra da Canastra, em Delfinópolis, Minas Gerais. Entre as espécies de lagarto registradas, Ameiva ameiva, Mabuya frenata, Tropidurus itambere, T. torquatus e Tupinambis merianae ocorrem em áreas abertas. Os espécimes de $A$. ameiva foram registrados nos diferentes hábitats em áreas cultivadas na área rural, e os de $M$. frenata predominaram em edificações humanas na área urbana.

O primeiro registro de Mabuya frenata para o Estado de Minas foi feito por Recoder \& Nogueira (2007) na porção sul do Parque Nacional Grande Sertão Veredas e, posteriormente, Costa et al. (2008) ampliaram sua distribuição para várias regiões do Estado, registrando sua ocorrência em áreas abertas do Cerrado, Mata Atlântica e zonas de transição. Costa et al. (2009), a partir de dados de coleção, também registraram a presença de várias espécies de lagartos para o município de Viçosa, incluindo Mabuya frenata, Hemidactylus mabouia e Tupinambis merianae e o anfisbenídeo Amphisbaena microcephala.

Ameiva ameiva é uma espécie de ampla distribuição na América do Sul, habitando desde ambientes xéricos como na Caatinga até ambientes extremamente úmidos na Amazônia (Vitt \& Colli 1994). 
Tabela 1. Número e porcentagem total de espécies de répteis registradas nas diferentes fisionomias do município de Ritápolis, Estado de Minas Gerais: Área antrópica (ARA); Área brejosa (ABA); Borda da mata (BMA); Campo cerrado (CCO); Campo rupestre (CRE); Mata de galeria (MGA).

Table 1. Number and percentage of reptile species recorded in the different areas of Ritápolis municipality, State of Minas Gerais: Anthropogenic area (ARA); Swamp area (ABA); Forest border (BMA); Cerrado field (CCO); Rocky field (CRE); Gallery forest (MGA).

\begin{tabular}{|c|c|c|c|}
\hline Táxon & Número & $\%$ & Hábitat \\
\hline \multicolumn{4}{|l|}{ TESTUDINES } \\
\hline \multicolumn{4}{|l|}{ Família Chelidae (2) } \\
\hline Acanthochelys radiolata (Mikan, 1820) & 1 & 0,8 & $\mathrm{ABA}$ \\
\hline Hydromedusa tectifera Cope, 1869 & 1 & 0,8 & $\mathrm{ABA}$ \\
\hline \multicolumn{4}{|l|}{ SQUAMATA: SAURIA } \\
\hline \multicolumn{4}{|l|}{ Família Anguidae (1) } \\
\hline Ophiodes striatus (Spix, 1824) & 4 & 3,1 & ARA \\
\hline \multicolumn{4}{|l|}{ Familia Gekkonidae (1) } \\
\hline Hemidactylus mabouia (Moreau de Jonnès, 1818) & 2 & 1,6 & ARA \\
\hline \multicolumn{4}{|l|}{ Família Gymnophthalmidae(1) } \\
\hline Cercosaura ocellata Wagler, 1830 & 1 & 0,8 & $\mathrm{CCO}$ \\
\hline \multicolumn{4}{|l|}{ Família Leiosauridae (1) } \\
\hline Enyalius bilineatus Duméril \& Bibron, 1837 & 16 & 12,4 & $\mathrm{CCO}, \mathrm{MGA}$ \\
\hline \multicolumn{4}{|l|}{ Familia Polychrotidae (1) } \\
\hline Polychrus acutirostris Spix, 1825 & 2 & 1,6 & MGA \\
\hline \multicolumn{4}{|l|}{ Família Scincidae (1) } \\
\hline Mabuya frenata (Cope, 1862) & 3 & 2,3 & ARA, CRE \\
\hline \multicolumn{4}{|l|}{ Familia Teiidae (2) } \\
\hline Ameiva ameiva (Linnaeus, 1758) & 10 & 7,8 & $\mathrm{CCO}, \mathrm{ARA}$ \\
\hline Tupinambis merianae (Duméril \& Bibron, 1839) & 1 & 0,8 & $\mathrm{CCO}$ \\
\hline \multicolumn{4}{|l|}{ Família Tropiduridae (1) } \\
\hline Tropidurus itambere Rodrigues, 1987 & 2 & 1,6 & ARA, CRE \\
\hline \multicolumn{4}{|l|}{ SQUAMATA: AMPHISBAENIA } \\
\hline \multicolumn{4}{|l|}{ Familia Amphisbaenidae (2) } \\
\hline Amphisbaena dubia L. Müller, 1924 & 1 & 0,8 & MGA \\
\hline Amphisbaena microcephala (Wagler, 1824) & 8 & 6,2 & $\mathrm{CCO}, \mathrm{MGA}$ \\
\hline \multicolumn{4}{|l|}{ SQUAMATA: SERPENTES } \\
\hline \multicolumn{4}{|l|}{ Familia Boidae (1) } \\
\hline Epicrates crassus Cope, 1862 & 3 & 2,3 & MGA, ARA \\
\hline \multicolumn{4}{|l|}{ Familia Dipsadidae (13) } \\
\hline Apostolepis assimilis (Reinhardt, 1861) & 1 & 0,8 & BMA \\
\hline Atractus pantostictus Fernandes \& Puorto, 1993 & 16 & 12,4 & ARA \\
\hline Erythrolamprus aesculapii (Linnaeus, 1766) & 1 & 0,8 & $\mathrm{CCO}$ \\
\hline Leptodeira annulata (Linnaeus, 1758) & 2 & 1,6 & $\mathrm{CCO}, \mathrm{ARA}$ \\
\hline Liophis poecilogyrus (Wied, 1825) & 2 & 1,6 & MGA \\
\hline Liophis typhlus (Linnaeus, 1758) & 1 & 0,8 & MGA \\
\hline Oxyrhopus guibei Hoge \& Romano, 1978 & 7 & 5,4 & ARA, BMA \\
\hline Philodryas olfersii (Lichtenstein, 1823) & 3 & 2,3 & ARA, BMA \\
\hline Pseudoboa nigra (Duméril, Bibron \& Duméril, 1854) & 1 & 0,8 & ARA, BMA \\
\hline Pseudoboa serrana Morato, Moura-Leite, Prudente \& Bérnils, 1995 & 1 & 0,8 & ARA \\
\hline Sibynomorphus mikanii (Schlegel, 1837) & 23 & 17,8 & ARA, BMA \\
\hline Sibynomorphus neuwiedi (Ihering, 1911) & 2 & 1,6 & BMA \\
\hline Xenodon merremii (Wagler, 1824) & 5 & 3,9 & ARA, BMA \\
\hline \multicolumn{4}{|l|}{ Familia Elapidae (1) } \\
\hline Micrurus frontalis (Duméril, Bibron \& Duméril, 1854) & 1 & 0,8 & $\mathrm{CCO}$ \\
\hline \multicolumn{4}{|l|}{ Familia Viperidae (3) } \\
\hline Bothropoides jararaca (Wied, 1824) & 2 & 1,6 & ARA, MGA \\
\hline Bothropoides neuwiedi (Wagler, 1824) & 3 & 2,3 & $\mathrm{CCO}$ \\
\hline Caudisona durissa (Linnaeus, 1758) & 3 & 2,3 & ARA, CCO \\
\hline Total & 129 & 100 & \\
\hline
\end{tabular}


Tupinambis merianae é uma espécie de lagarto de grande porte que ocorre do norte da Argentina até o sul da Amazônia (Vanzolini et al. 1980) e está listado como vulnerável no Apêndice II da CITES por ser uma espécie muito visada por caçadores, o que se pode confirmar para essa região.

Os lagartos do gênero Tropidurus são extremamente abundantes nos vários tipos de formações abertas e ocorrem na América do Sul continental a leste e a oeste dos Andes e nas ilhas Galápagos (Rodrigues 1987). Dentro da área de estudo, encontra-se T. itambere (Figura 6d), registrado em Minas Gerais por Recoder \& Nogueira (2007) em área de Cerrado. Cercosaura ocellata também é uma espécie encontrada no Cerrado e em algumas savanas, ao leste da Amazônia. No Brasil é encontrada no Acre, Amapá, Maranhão, Pará, Roraima, Bahia, Rio Grande do Sul, São Paulo (Vitt \& Colli 1994) e em Minas Gerais (Recoder \& Nogueira, 2007).

Entre as espécies de lagartos do gênero Enyalius, E. bilineatus é uma das espécies habitantes de regiões florestadas, encontrada principalmente nas matas ciliares associadas à Mata Atlântica do sudeste do Brasil (Jackson 1978) ou mesmo no Cerrado do Brasil Central (Nogueira 2001, Zatz 2002). Em Minas Gerais a espécie foi registrada em matas preservadas da Estação Ambiental de Peti por Bertoluci et al. (2009) e em Viçosa em áreas antropizadas e em matas em regeneração ou secundárias por Costa et al. (2009). No entanto, o padrão cromático de alguns indivíduos capturados em matas de galeria na área de Cerrado, também considerados como E. bilineatus no presente estudo, não corresponde aos demais listados para a espécie. Talvez um estudo de revisão do gênero Enyalius poderá demonstrar a existência de uma nova espécie para esta região.

O lagarto Polychrus acutirostris (Figura 5f) é uma espécie arborícola de hábitos letárgicos e que ocorre em todos os tipos de cerrado e caatinga onde existe um estrato arbóreo, podendo às vezes ser encontrada em arbustos baixos e até no chão (Vitt \& Lacher, 1981). O único exemplar capturado no presente estudo encontrava-se sobre um arbusto na área de trabalho.

A busca ativa permitiu amostrar espécies de serpentes de grande porte, como foi o caso de Epicrates crassus. O complexo Epicrates cenchria é endêmico da região Neotropical, ocorrendo em porções continentais da América Central e do Sul no Bioma Mata Atlântica em toda a América do Sul, com registros também para a Floresta Amazônica. O status taxonômico das nove subespécies atualmente reconhecidas foi avaliado com base na morfologia externa, osteologia e caracteres do hemipênis. Os resultados obtidos através de análises quantitativas e qualitativas apoiam o reconhecimento de E. alvarezi, E. assis, E. cenchria, E. crassus e E. maurus como espécies distintas (Passos \& Fernandes 2008). Segundo registros de distribuição, a espécie que ocorre na área de coleta do presente estudo seria E. crassus. Normalmente os espécimes são mortos pelos proprietários rurais, o que pode representar um grande impacto sobre as populações desta espécie. Palmuti et al. (2009) encontraram um espinho de porcoespinho (Coendu sp.) no conteúdo estomacal de um indivíduo dessa espécie coletado no município de Caratinga (MG).

Habitante dos biomas Amazônia, Caatinga, Cerrado, Mata Atlântica e Pantanal, Leptodeira annulata distribui-se por várias regiões do Brasil, especialmente pela região litorânea, da Bahia até São Paulo (Freitas 2003) e no Parque Nacional das Emas, em Goiás (Valdujo et al. 2009). Em Minas Gerais, o local mais próximo de nossa área de estudo em que essa serpente foi registrada é a Estação Ambiental de Peti, localizada nos municípios de São Gonçalo do Rio Abaixo e de Santa Bárbara, por Bertoluci et al. (2009). É uma serpente noturna, encontrada em florestas primárias e secundárias, mas pode ser encontrada próxima a áreas antropizadas e sua densidade pode se acentuar em áreas com altas densidades de anuros (Vitt 1996).
No Brasil, Apostolepis assimilis (Figura 6h) é encontrada no Distrito Federal, Espírito Santo, Goiás, Mato Grosso, Mato Grosso do Sul, Minas Gerais, Paraíba, Paraná, Pernambuco, São Paulo e Tocantins (Ferrarezzi et al. 2005, São Pedro \& Pires 2009, Valdujo et al. 2009), onde habita biomas como a Mata Atlântica e o Cerrado, podendo ocorrer inclusive em áreas urbanas (Ferrarezzi 1993, Ferrarezzi et al. 2005). A. assimilis pode possuir hábitos subterrâneos ou fossoriais, e se alimentar de pequenos anfisbenídeos e de serpentes fossórias do grupo Scolecophidia (Ferrarezzi 1993, Ferrarezzi et al. 2005).

Micrurus frontalis é a coral verdadeira mais abundante e amplamente distribuída nas áreas de Cerrado (Mesquita et al. 2007, Sawaya et al. 2008, São Pedro \& Pires 2009). É a espécie mais amplamente distribuída de todos os membros do complexo M. frontalis, ocorrendo desde as formações de Cerrado do Brasil Central (inclusive os Estados de São Paulo e Minas Gerais) para o Oeste até a fronteira com a Bolívia, e nos estados brasileiros de Mato Grosso e Mato Grosso do Sul. Ao leste, aparentemente atinge a costa Atlântica somente no Estado do Espírito Santo devido à formação montanhosa da Serra do Mar ao sul. Na direção Sul-Sudoeste ela alcança o Paraguai onde está restrita possivelmente pelo Rio Paraná e o Rio Paraguai (Silva Jr. \& Sites Jr. 1999).

A (jararaca-do-rabo-branco, Bothropoides neuwiedi (Figura 8a), ocorre desde áreas do extremo nordeste do Brasil e do Estado de Rondônia até o Uruguai e região central da Argentina, nas quais diversas subespécies foram reconhecidas por Campbell \& Lamar (1989). Na revisão taxonômica desse complexo, Silva \& Rodrigues (2008) detectaram que B. neuwiedi (considerando as 12 subespécies) é, na verdade, formado por sete espécies plenas. Pelo grande número de registros, aparentemente, B. neuwiedi é mais comum do que Bothropoides jararaca na área de estudo, considerando que grande parte das espécies do grupo neuwiedi é típica de áreas abertas (Valdujo et al. 2002), ao passo que B. jararaca ocorre primordialmente em áreas florestadas (Martins et al. 2001).

As espécies Philodryas olfersii (Figura 6d) e B. jararaca, registradas no presente estudo, foram também registradas na Mata Atlântica por Hartmann et al. (2009a), no Parque Estadual da Serra do Mar, litoral Norte do Estado de São Paulo, e por Bertoluci et al. (2009) em área de transição entre Mata Atlântica e Cerrado no Estado de Minas Gerais, embora $P$. olfersii seja uma espécie que também possa ocorrer em áreas alteradas (Hartmann \& Marques 2005). Também na Mata Atlântica, juntamente com outras espécies de serpentes, Liophis poecilogyrus (Figura 7b) foi registrada por Marques et al. (2001) e Gonçalves et al. (2007). Bertoluci et al. (2009) confirmaram a sua ocorrência para Minas Gerais.

Várias espécies de serpentes registradas no presente estudo também foram registradas por São Pedro \& Pires (2009) na região de Ouro Branco, Minas Gerais, numa área de transição entre Mata Atlântica e Cerrado, exceto Epicrates crassus, Leptodeira annulata, Liophis typhlus (Figura 7c), Pseudoboa nigra (Figura 7g), P. serrana (Figura 7h) e Sibynomorphus neuwiedi. No entanto, Recoder \& Nogueira (2007), no trabalho que apresenta as espécies de répteis da porção sul do Parque Nacional Grande Sertão Veredas, no Estado de Minas Gerais, incluem E. crassus, L. typhlus e P. nigra dentre as espécies registradas. Em áreas de Mata Atlântica no Estado de Minas Gerais foi registrada L. annulata por Bertoluci et al. (2009), P. serrana por Morato et al. (1995) e S. neuwiedi por Palmuti et al. (2009).

Oxyrhopus guibei $\mathrm{e}$ S. mikanii foram as espécies mais comumente registradas na área urbana do município e Erythrolamprus aesculapii e S. neuwiedi apresentaram baixa ocorrência e foram registradas apenas na área rural. E. aesculapii é uma falsa-coral que apresenta ampla distribuição na região neotropical e no Brasil ocorre desde a Amazônia até a região sul, sendo bastante comum na Mata Atlântica do Sudeste 
(Marques 1996), da mesma forma que S. neuwiedi (Marques et al. 2001). Sibynomorphus mikanii e O. guibei foram registrada por Costa et al. (2007) e Valdujo et al. (2009) em regiões de Cerrado. O fato de $O$. guibei ser considerada um mímico de $M$. frontalis pode possibilitar o favorecimento desta espécie (Sousa \& Hudson 2005).

Próximo à área de estudo encontra-se a FLONA de Ritápolis, que ocupa uma área de 89,50 ha, sendo composta por vegetação típica dos biomas Mata Atlântica e Cerrado. Para o meio científico, o desenvolvimento do presente estudo possibilitou a identificação de locais de ocorrência dos grupos-chave de répteis para realização de futuras pesquisas no ecótono Mata Atlântica-Cerrado. Considerando-se apenas os resultados do presente trabalho, a riqueza de répteis pode ser considerada baixa se comparada à de outras localidades do Estado de Minas Gerais (e.g. Recoder \& Nogueira 2007, Bertoluci et al. 2009, Costa et al. 2009, Palmuti et al. 2009 e São Pedro \& Pires 2009). Entretanto, as ocorrências previamente registradas de A. dubia (Barros Filho et al. 2005) e H. tectifera (Sousa \& Novelli 2009) no Estado de Minas Gerais indicam a grande potencialidade do Campo das Vertentes em revelar a ocorrência de espécies novas ou a ampliação na distribuição de outras. Tal fato aumenta a necessidade de preservação da região que sofre uma pressão crescente de fragmentação em uma área de transição entre dois hotspots de diversidade.

\section{Agradecimentos}

B. M. Sousa agradece à Fundação de Amparo à Pesquisa do Estado de Minas Gerais (FAPEMIG) pelo financiamento do projeto "Répteis em fragmentos de Cerrado do Campo das Vertentes, MG" (EDT-230/05). Recursos adicionais não financiados foram providos pelo Departamento de Zoologia da Universidade Federal de Juiz de Fora. Agradecemos ao Instituto Brasileiro do Meio Ambiente e dos Recursos Naturais Renováveis (IBAMA) pela permissão para coleta e transporte dos espécimes (02015.010660/05-88). Agradecemos também aos pesquisadores do Museu Nacional do Rio de Janeiro, especialmente Ronaldo Fernandes e Renato Bérnils, e a Miguel Trefaut Urbano Rodrigues, do Museu da Universidade de São Paulo pela ajuda na identificação de algumas espécies. Agradecemos ao Fabiano Matos Vieira pela ajuda na elaboração das figuras. Ao Senhor Sebastião Higino de Souza pela permissão de desenvolver esta pesquisa em sua propriedade e ao Prefeito do município de Ritápolis, Higino Zacarias e Souza e Maria Rita de Souza Napoleão, Secretária de Educação, pelo apoio logístico nas pesquisas desenvolvidas nos períodos de 04 a 08 de dezembro de 2002 e de 13 a 15 de dezembro de 2003. Agradecemos também à Maria de Nazaré Serpa, ao Antonio Luiz da Silva (Antônio da Célia) e ao Gilberto Gregório de Sousa pela valiosa ajuda nos trabalhos de campo.

\section{Referências Bibliográficas}

ASSIS, V.B. 1999. Introdução às serpentes da "Serra do Cipó" (municípios de Santana do Riacho e Jaboticatubas). Bios. 7(7):69-71.

BARROS FILHO, J.D., SOUSA, B.M. \& CARVALHO, R.M.H. 2005 Amphisbaena dubia (NCN). Herpetol. Rev. 36(3):335.

BERNILS, R.S., NOGUEIRA, C.C. \& XAVIER-DA-SILVA, V. 2009. Répteis. In Biota Minas: diagnóstico do conhecimento sobre a biodiversidade no Estado de Minas Gerais (G. M. Drummond, C.S. Martins, M.B. Greco \& F. Vieira, org.). Fundação Biodiversitas, Belo Horizonte, p.251-278.

BÉRNILS, R.S. 2010. Brazilian reptiles: list of species. http://www. sbherpetologia.org.br/ (último acesso em 12/05/2010).

BERTOLUCI, J. 1998. Serpentes. In Livro Vermelho das Espécies Ameaçadas de Extinção da Fauna de Minas Gerais (A.B.M. Machado, G.A. Fonseca, L.M.S. Aguiar \& L.V. Lins, eds.). Fundação Biodiversitas, Belo Horizonte, p.417.

BERTOLUCI, J., CANELAS, M.A.S., EISEMBERG, C.C., PALMUTI, C.F.S \& MONTINGELLI, G.G. 2009. Herpetofauna of Estação Ambiental de
Peti, an Atlantic Rainforest fragment of Minas Gerais State, southeastern Brazil. Biota Neotrop. 9(1): http://www.biotaneotropica.org.br/v9n1/en/a bstract?inventory+BN01409012009 (último acesso em 22/04/2010).

BONIN, F., DEVAUX, B. \& DUPRE, A. 2006. Turtles of the World. A and C Black Publishers, London.

BRITES, V.L.C. \& BAUAB, F.A. 1988. Fauna ofidiana do município de Uberlândia, Minas Gerais - Brasil. I. Ocorrência na área urbana. Rev. Cent. Cienc. Biomed. Univ. Fed. Uberlandia 4(1):3-8.

CAMPBELL, J.A. \& LAMAR, W.W. 1989. The Venomous Reptiles of the Latin America. Cornell University Press, Ithaca.

CECHIN, S.T.Z. 1999. História natural de uma comunidade de serpentes na região da Depressão Central (Santa Maria), Rio Grande do Sul, Brasil. Tese de Doutorado, Pontifícia Universidade Católica do Rio Grande do Sul, Porto Alegre.

CECHIN, S.Z. \& MARTINS, M. 2000. Eficiência de armadilhas de queda (pitfall traps) em amostragens de anfíbios e répteis no Brasil. Rev. Bras. Zool. 17(3):729-740.

COLLI, G.R., BASTOS, R.P. \& ARAÚJO, A.F.B. 2002. The character and dynamics of the Cerrado herpetofauna. In The Cerrados of Brazil: ecology and natural history of a Neotropical Savanna (P.S. Oliveira \& R.J. Marquis, eds.). Columbia University Press, New York, p. 223-241.

COLWELL, R.K. 2006. Estimates: statistical estimation of species richness and shared species from samples. Version 8. http://purl.oclc.org/estimates (último acesso em 06/05/2010).

Minas Gerais. Conselho Estadual de Política Ambiental - COPAM. 2008. Deliberação COPAM n ${ }^{\circ} 366,15$ de dezembro de 2008. Aprova a lista de espécies ameaçadas de extinção da fauna do Estado de Minas. http:// www.ief.mg.gov.br/images/stories/biodiversidade/faunaameacada dn366_2008.pdf (último acesso em 01/07/2009).

COSTA, G.C., NOGUEIRA, C., MACHADO, R.B. \& COLLI, G.R. 2007. Squamate richness in the Brazilian Cerrado and its environmental climatic associations. Divers Distrib. 13(6):714-724.

COSTA, H.C., FERNANDES, V.D., VRCIBRADIC, D. \& FEIO, R.N. 2008. Notes on Geographic Distribution: Reptilia, Scincidae, Mabuya frenata: Distribution extension. Check List 4(1):86-88.

COSTA, H.C., FERNANDES, V.D., RODRIGUES, A.C. \& FEIO, R.N. 2009. Lizards and Amphisbaenians, municipality of Viçosa, state of Minas Gerais, southeastern Brazil. Check List 5(3):732-745.

DI-BERNARDO, M. 1998. História natural de uma comunidade de serpentes da borda oriental do Planalto das Araucárias, Rio Grande do Sul, Brasil. Tese de Doutorado, Universidade Estadual Paulista, Rio Claro.

DRUMMOND, G.M., MARTINS, C.S., MACHADO, A.B.M., SEBAIO, F.A \& ANTONINI, Y. 2005. Biodiversidade em Minas Gerais: um Atlas para sua conservação. 2 ed. Fundação Biodiversitas, Belo Horizonte.

ERNST, C.H. \& BARBOUR, R.W. 1989. Turtles of the world. Smithsonian Institution Press, Washington, DC.

FEIO, R.N. \& CARAMASCHI, U. 2002. Contribuição ao conhecimento da herpetofauna do nordeste do estado de Minas Gerais, Brasil. Phyllomedusa 1(2):105-111.

FERRAREZZI, H. 1993. Sistemática filogenética de Elapomorphus, Phalotris e Apostolepis (Serpentes: Colubridae: Xenodontinae). Dissertação de Mestrado, Universidade de São Paulo, São Paulo.

FERRAREZZI, H., BARBO, F.E. \& ALBUQUERQUE, C.E. 2005. Phylogenetic relationships of a new species of Apostolepis from braziliam cerrado with notes on the assimilis group (Serpentes: Colubridae: Xenodontinae: Elapomorphini). Pap. Avulsos Zool. 45(16):215-229.

FREITAS, M.A. 2003. Serpentes brasileiras. Edição do Autor, Lauro de Freitas.

FRITZ, U. \& HAVAS, P. 2007. Checklist of Chelonians of the World. Vertebr. Zool. 57(2):149-368.

GIBBONS, J.W., SCOTT, D.E., RYAN, T.J., BUHLMANN, K.A., TUBERVILLE, T.D., METTS, B.S., GREENE, J.L., MILLS, T., LEIDEN, Y., POPPY, S. \& WINNE, C.T. 2000. Reptiles in decline: The global decline of reptiles, déjà vu amphibians. BioScience 50(8):653666.

GONÇALVES, M.A.P.L., AGUIAR, F.V.O., CAMARGO, J.V.C., BARROS FILHO, J.D. \& CARVALHO E SILVA, S.P. 2007. Levantamento preliminar da fauna de répteis do Parque Nacional Serra dos Órgãos. In 
Ciência e Conservação na Serra dos órgãos (C. Cronemberger \& E.B. Viveiros de Castro, eds.). Ibama, Brasília, DF, p.1-17.

HARTMANN, P.A. \& MARQUES, O.A.V. 2005. Diet and habitat use of two sympatric species of Philodryas (Colubridae), in South Brazil. AmphibiaReptilia 26(1):25-31.

HARTMANN, P.A., HARTMANN, M.T. \& MARTINS, M. 2009a. Ecology of a snake assemblage in the Atlantic Forest of southeastern Brazil. Pap. Avulsos Zool. 49(27):343-360.

HARTMANN, P.A., HARTMANN, M.T. \& MARTINS, M. 2009b. Ecologia e história natural de uma taxocenose de serpentes no Núcleo Santa Virgínia do Parque Estadual da Serra do Mar, no sudeste do Brasil. Biota Neotrop. 9(3): http://www.biotaneotropica.org.br/v9n3/en/ abstract?article+BN03609032009 (ultimo acesso em 22/04/2010).

JACKSON, J.F. 1978. Differentiation in the genera Enyalius and Strobilurus (Iguanidae): implications for pleistocene climatic changes in eastern Brazil. Arq. Zool. 30(1):1-79.

MACHADO, A.B.M., DRUMMOND, G.M. \& PAGLIA, A.P. 2008. Livro vermelho da fauna brasileira ameaçada de extinção. 1 ed. Fundação Biodiversitas, Belo Horizonte.

MARQUES, O.A.V. 1996. Biologia reprodutiva da cobra-coral Erythrolamprus aesculapii Linnaeus (Colubridae), no sudeste do Brasil. Rev. Bras. Zool. 13(3):747-753.

MARQUES, O.A.V., ETEROVIC, A. \& SAZIMA, I. 2001. Serpentes da Mata Atlântica. Guia ilustrado para a Serra do Mar. Holos, Ribeirão Preto.

MARQUES, O.A.V. \& SAZIMA, I. 2004. História natural dos répteis da Estação Ecológica Juréia-Itatins. In Estação Ecológica Juréia-Itatins: ambiente físico, flora e fauna (O.A.V. Marques \& W. Duleba, eds.). Holos, Ribeirão Preto, p. 257-277.

MARTINS, M., ARAÚJO, M.S., SAWAYA, R.J. \& NUNES, R. 2001. Diversity and evolution of macrohabitat use body size and morphology in a monophyletic group of neotropical pitvipers (Bothrops). J. Zool. 254(4):529-538.

MESQUITA, D.O., COLLI, G.R. \& VITT, L.J. 2007. Ecological release in lizard assemblages of Neotropical savannas. Oecologia 153(1):185-195

MITTERMEIER, R.A., GIL, P.R., HOFMANN, M., PILGRIM, J., BROOKS, T., MITTERMEIER, C.G., LAMOREAUX, J. \& FONSECA, G.A.B. 2004. Hotspots revisited: Earth's biologically richest and most endangered terrestrial ecoregions. Cemex, Washington, DC.

MORATO, S.A.A., MOURA-LEITE, J.C., PRUDENTE, A.L.C. \& BERNILS, R.S. 1995. A new species of Pseudoboa Schneider, 1801 from Southeastern Brazil (Serpentes: Colubridae: Xenodontinae: Pseudoboini). Biociências 3(2):253-264.

NOGUEIRA, C.C. 2001. New records of Squamate Reptiles in Central Brazilian Cerrado II: Brasília Region. Herpetol. Rev. 32(4):285-287.

NOGUEIRA, C., COLLI, G.R. \& MARTINS, M. 2009. Local richness and distribution of the lizard fauna in natural habitat mosaics of the Brazilian Cerrado. Austral Ecol. 34(1):83-96.

PALMUTI, C.F.S., CASSIMIRO, J. \& BERTOLUCI, J. 2009. Food habits of snakes from the RPPNFeliciano Miguel Abdala, an Atlantic Forest fragment of southeastern Brazil. Biota Neotrop. 9(1): http://www.biotaneotropica. org.br/v9n1/en/abstract?short-communication+BN02209012009 (último acesso em 22/04/2010).

PASSOS, P. \& FERNANDES, R. 2008. Revision of the Epicrates cenchria Complex (Serpentes: Boidae). Herpetol. Monogr. 22:1-30.

PEREZ, R. \& RIBEIRO, S.L.B. 2008. Reptilia, Squamata, Amphisbaenidae, Leposternon spp.: Distribution extension, new state record, and geographic distribution map. Check List 4(3):291-294.

Ritápolis. 2006. O Município de Ritápolis. Secretaria de Educação. p.1-6.

RECODER, R. \& NOGUEIRA, C. 2007. Composição e diversidade de répteis na região sul do Parque Nacional Grande Sertão Veredas, Brasil Central. Biota Neotrop. 7(3): http://www.biotaneotropica.org.br/v7n3/pt/abstract ?inventory+BN01107032007 (último acesso em 10/05/2010).

RODRIGUES, M.T. 1987. Sistemática, ecologia e zoogeografia dos Tropidurus do grupo torquatus ao Sul do Rio Amazonas (Sauria, Iguanidae). Arq. Zool. 31(3):105-230.

RODRIGUES, M.T. 2005. Conservação dos répteis brasileiros: os desafios para um país megadiverso. Megadiversidade 1(1):87-94.
RÖMPP, O. 2008. Keeping and breeding the Brazilian Radiolated Swamp Turtle Acanthochelys radiolata (Mikan, 1820) with a discussion of primary and secondary diapause. Emys 15(2):4-15.

SÃO PEDRO, V.A. \& PIRES, M.R. 2009. As Serpentes da Região de Ouro Branco, extremo sul da Cadeia do Espinhaço, Minas Gerais. Revista Ceres 56(2):166-171.

SAWAYA, R.J., MARQUES, O.A.V. \& MARTINS, M. 2008. Composição e história natural das serpentes de Cerrado de Itirapina, São Paulo, sudeste do Brasil. 8(2): http://www.biotaneotropica.org.br/v8n2/en/abstract?inve ntory+BN01308022008 (último acesso em 10/05/2010).

SAZIMA, I. \& HADDAD, C.F.B. 1992. Répteis da Serra do Japi: notas sobre história natural. In História Natural da Serra do Japi: ecologia e preservação de uma área florestal no sudeste do Brasil (L.P.C. Morellato, ed.). Editora da Unicamp, Campinas. p. 212-237.

SILVA Jr., N.J. \& SITES JR., J.W. 1999. Revision of the Micrurus Frontalis complex (Serpentes: Elapidae). Herpetol. Monogr. 13:142-194.

SILVA, V.X. \& RODRIGUES, M.T. 2008. Taxonomic revision of the Bothrops neuwiedi complex (Serpentes, Viperidae) with description of a new species. Phyllomedusa 7(1):45-90.

SOUSA, B.M. \& HUDSON, A.A. 2005. O mimetismo em função da vida: o caso de Oxyrhopus guibei (Serpente: Colubridae) no município de Ritápolis, Minas Gerais. An. de Etologia 23(1):90-91.

SOUSA, B.M. \& NOVELLI, I.A. 2009. Reptilia, Testudines, Chelidae: Hydromedusa tectifera: Distribution extension in Brazil. Check List 5(3):396-398.

SOUZA, F.L. 2005. Geographical distribution patterns of South American side-necked turtles (Chelidae), with emphasis on Brazilian species. Rev. Esp. Herp. 19:33-46.

STRÜSSMANN, C. \& SAZIMA, I. 1993. The snake assemblage of the pantanal at Poconé, western Brazil: faunal composition and ecological summary. Stud. Neotrop. Fauna and Environment. 28(3):157-168.

UETZ, P. \& HALLERMANN, J. 2010. The JCVI/TIGR Reptile Database. http://www.reptiledatabase.org (último acesso em 12/01/2010).

VALDUJO, P.H., NOGUEIRA, C.C. \& MARTINS, M. 2002. Ecology of Bothrops neuwiedi pauloensis (Serpentes: Viperidae: Crotalinae) in the Brazilian Cerrado. J. Herpetol. 36(2):169-176.

VALDUJO, P.H., NOGUEIRA, C.C., BAUMGARTEN, L., RODRIGUES, F.H.G., ELETROVIC, A., RAMO-NETO, M.B. \& MARQUES, O.A.V. 2009. Squamate Reptiles from Parque Nacional das Emas and surroundings, Cerrado of Central Brazil. Check List 5(3):405-417.

VANZOLINI, P.E., RAMOS-COSTA, A.M.M. \& VITT, L.J. 1980. Répteis das Caatingas. Academia Brasileira de Ciências, Rio de Janeiro.

VITT, L.J. \& COLLI, G.R. 1994. Geographical ecology of a neotropical lizard: Ameiva ameiva (Teiidae) in Brazil. Can. J. Zool. 72(11):1986-2008.

VITT, L.J. \& LACHER, T.E. 1981. Behaviour, habitat, diet and reproduction of the iguanid lizard Polychrus acutirostris in the Caatinga of northeastern Brazil. Herpetologica 37(1):53-63.

VITT, L.J. 1996. Ecological observations on the tropical colubrid snake Leptodeira annulata. Herpetol. Nat. Hist. 4(1):69-76.

VITT, L.J., CALDWELL, J.P., COLLI, G.R., GARDA, A.A., MESQUITA, D.O., FRANÇA, F.G.R. \& BALBINO, S.F. 2002. Um guia fotográfico dos répteis e anfíbios da região do Jalapão no Cerrado brasileiro. Special Publications in Herpetology, Sam Noble Oklahoma Museum of Natural History 1:1-17.

ZANELLA, N. 2004. História natural de uma comunidade de serpentes no Planalto médio, Rio Grande do Sul, Brasil. Tese de doutorado, Pontifícia Universidade Católica do Rio Grande do Sul, Porto Alegre.

ZATZ, M.G. 2002. O polimorfismo cromático e sua manutenção em Enyalius sp (Squamata: Leiosauridae) no Cerrado do Brasil Central. Dissertação de Mestrado, Universidade de Brasília, Brasília, DF. 